\title{
Analysis and suppression of thermal effect of an ultra-stable laser interferometer for space-based gravitational waves detection
}

\author{
Guanfang Wang (王观芳) ${ }^{1}$, Zhu Li (李 祝) ${ }^{1 *}$, Jialing Huang (黄家玲 $)^{2}$, Huizong Duan (段会宗) ${ }^{1}$, Xiangqing Huang (黄祥青) ${ }^{1}$ ， \\ Hongfan Liu (刘洪凡) ${ }^{1}$, Qi Liu (刘 褀) ${ }^{1}$, Shanqing Yang (杨山清) ${ }^{1}$, Liangcheng Tu (涂良成) ${ }^{1}$, and Hsien-Chi Yeh (叶贤基) ${ }^{1}$ \\ ${ }^{1}$ MOE Key Laboratory of TianQin Mission, TianQin Research Center for Gravitational Physics \& School of Physics and Astronomy, Frontiers Science Center \\ for TianQin, CNSA Research Center for Gravitational Waves, Sun Yat-sen University (Zhuhai Campus), Zhuhai 519082, China \\ ${ }^{2}$ Shenhe Middle School, Heyuan 517000, China
}

*Corresponding author: lizhu@mail.sysu.edu.cn

Received September 10, 2021 | Accepted September 30, 2021 | Posted Online October 25, 2021

\begin{abstract}
In this paper, we present a suppression method for the thermal drift of an ultra-stable laser interferometer. The detailed analysis on the Michelson interferometer indicates that the change in optical path length induced by temperature variation can be effectively reduced by choosing proper thickness and/or incident angle of a compensator. Taking the optical bench of the Laser Interferometer Space Antenna Pathfinder as an example, we analyze the optical bench model with a compensator and show that the temperature coefficient of this laser interferometer can be reduced down to $1 \mathrm{pm} / \mathrm{K}$ with an incident angle of $0.267828 \mathrm{rad}$. The method presented in this paper can be used in the design of ultra-stable laser interferometers, especially for space-based gravitational waves detection.
\end{abstract}

Keywords: gravitational waves detection; laser interferometer; temperature compensation; optical path calculation. DOI: 10.3788/COL202220.011203

\section{Introduction}

The gravitational wave (GW) was a great prediction of Einstein's general theory of relativity 100 years ago. Nowadays, the detection of GWs has been a challenge and also an opportunity for opening a new window to observe our universe $e^{[1-3]}$. On September 14, 2015, the first direct detection of a GW signal GW150914 made by the Laser Interferometer GW Observatory (LIGO) successfully confirmed the existence of $\mathrm{GWs}^{[4-6]}$. In order to obtain more abundant GW signals in the lower frequency region, European Space Agency (ESA) and National Aeronautics and Space Administration (NASA) jointly proposed the Laser Interferometer Space Antenna (LISA) project in the $1990 \mathrm{~s}^{[7]}$. LISA is a space-based GW observatory, which is to form a regular triangle constellation operating in solar orbit. The satellites are connected by laser links so that a large laser interferometer in space can be constructed ${ }^{[8-10]}$. This long interferometer armlength allows the space-based GW observatory to detect the signals in a frequency range of $10^{-4}-1 \mathrm{~Hz}^{[11]}$. The TianQin project orbiting the earth and the Taiji project orbiting the sun proposed by Chinese researchers are also space-based GW detection missions, and the observatories will be launched in the next 10 to 15 years $^{[12-15]}$.
Laser interferometry is the core technology in space-based GW observatories. There are two polarization modes of GWs, which will make one interferometer armlength compressed and another orthogonal interferometer armlength stretched ${ }^{[16]}$. The laser interferometer can measure the optical-path-length difference between two arms so that it is an ideal measurement tool for detecting GWs ${ }^{[17]}$. Nevertheless, the space-based GW detection missions, such as LISA, TianQin, and Taiji, require laser interferometry to be extremely precise over arm-lengths of millions of kilometers in space. The identical satellites of LISA are $2.5 \times 10^{6} \mathrm{~km}$ apart, and the measurement noise of the interferometer is $18 \mathrm{pm} / \mathrm{Hz}^{1 / 2}$. The satellites of TianQin are $1.7 \times 10^{5} \mathrm{~km}$ apart, and the measurement noise is $1 \mathrm{pm} / \mathrm{Hz}^{1 / 2}$. The noise level of Taiji is $8 \mathrm{pm} / \mathrm{Hz}^{1 / 2}$ over an inter-satellite distance of $3 \times 10^{6} \mathrm{~km}$. The measurement frequency band is about $10^{-4} \mathrm{~Hz}$ to $1 \mathrm{~Hz}^{[18-20]}$. Achieving such high performance of laser interferometry in space is a huge challenge, and the performance of laser interferometry directly determines the sensitivity of space-based GW detection.

It is critical to evaluate the influence of environmental noises in high precision laser interferometry. In the LIGO GW detector, a temperature control system with a noise level of 
micro-Kelvin $(\mu \mathrm{K})$ has been used to reduce the measurement noise caused by temperature fluctuation, and the whole device was placed in vacuum chambers ${ }^{[21,22]}$. However, in the spacebased GW observatory, the space environmental factors, such as temperature variation and spacecraft vibration, will induce non-negligible measurement errors of laser interferometry. For this reason, researchers have developed an ultra-stable laser interferometer which is made of an ultra-low expansion glass (ULE) or Zerodur substrate and fused-silica-based optical components, and constructed by silicate bonding technique ${ }^{[23]}$. This type of ultra-stable laser interferometer has been verified onboard in LISA Pathfinder (LPF) and TianQin-1, which are the technology demonstration satellites for LISA and TianQin, respectively ${ }^{[24]}$. According to the in-orbit experimental results, the laser interferometer was still affected by temperature variation. As the temperature variation outside the satellites can be more than $100^{\circ} \mathrm{C}$, even the satellite has installed thermo-shields, so the temperature inside the satellite can be changed several degrees centigrade. This is not acceptable to the high precision laser interferometer with a picometer noise level.

The influence of temperature fluctuation on the laser interferometer system is very complicated, and there are many coupling factors. As the core unit of the interferometer system, the optical bench interferometer (OBI) is affected by temperature fluctuation mainly through two mechanisms. One is the thermal expansion of the lens, and the other is the change of the refractive index of the lens ${ }^{[25-27]}$. Both of them will lead to the variation in the optical path length of the laser interferometer. Based on these two coupling mechanisms, the optical path length coupling with the temperature fluctuation is analyzed in this paper, and an effective method for compensating the thermal effect is proposed. In Section 2, a typical Michelson interferometer is used as a simple case for analytical analysis. The compensation method is discussed in Section 3. The verification by finite element analysis shows that the thickness and the incident angle of the compensator affect the temperature coupling coefficient of the laser interferometer. In Section 4, a heterodyne laser interferometer model of LPF is used as an example for demonstration of the compensator design, and the design is verified by using finite element analysis ${ }^{[28,29]}$. In Section 5, we conclude that this compensation scheme can measure displacement used in the design of the laser interferometer for space GW detection in the future ${ }^{[30]}$.

\section{Analysis of Thermal Effect in the Basic Optical Path}

The Michelson laser interferometer is the most common one among various kinds of optical interferometers. A basic Michelson interferometer consists minimally of mirrors M1, M2 and a beam splitter (BS). In space GW detection, M1 and M2 can be regarded as the reflection surfaces of a pair of test masses. In Fig. 1, a laser source emits a laser beam that hits the BS. The BS is partially reflective, so a part of the laser is transmitted through to $\mathrm{M} 2$ in the direction of $\mathrm{B}$. The other part of the laser is reflected in the direction of A. Both beams recombine to

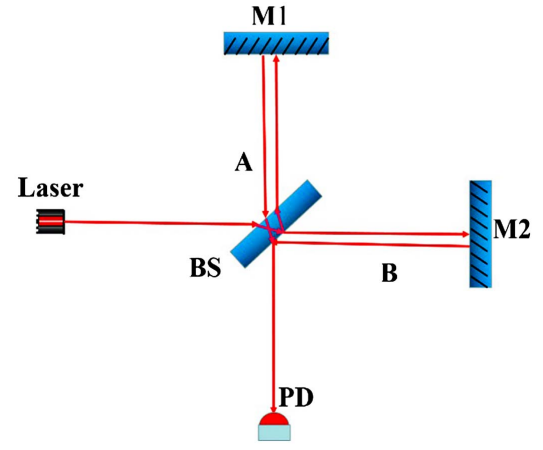

Fig. 1. Schematic diagram of the basic Michelson interferometer.

produce an interference pattern incident on the photo diode (PD). If a small displacement of M1 (or M2) changes the optical path of A (or B), the phase signal of the interference will change accordingly. The phase directly reflects the optical path variation $\Delta s$.

The optical path $s$ is related to the refractive index $n$ and the geometrical path length $l$; both are affected by temperature fluctuation, as shown in Eq. (1):

$$
s=n(T) l(T) .
$$

The thermal effects of light route A and light route B need to be analyzed, respectively.

The geometric path segments in light route $\mathrm{A}$ are set as $l_{i}$ $(i=1-6)$ in Fig. 2(a). The optical path of light path A can be written as

$$
\begin{aligned}
s_{A} & =l_{1}+n l_{2}+l_{3}+l_{4}+n l_{5}+l_{6} \\
& =l_{2}(n-1)+l_{5}(n-1)+l_{A 0},
\end{aligned}
$$

where $l_{A 0}=\sum_{i=1}^{6} l_{i}$ is the geometric path length of light route A. Thus, the coupling of temperature $\mathrm{d} T$ to the optical path $\mathrm{d} s_{A}$ can be expressed by

$$
\frac{s_{A}}{\mathrm{~d} T}=\frac{\mathrm{d} l_{2}(n-1)}{\mathrm{d} T}+\frac{\mathrm{d} l_{5}(n-1)}{\mathrm{d} T}+\frac{\mathrm{d} l_{A 0}}{\mathrm{~d} T}
$$

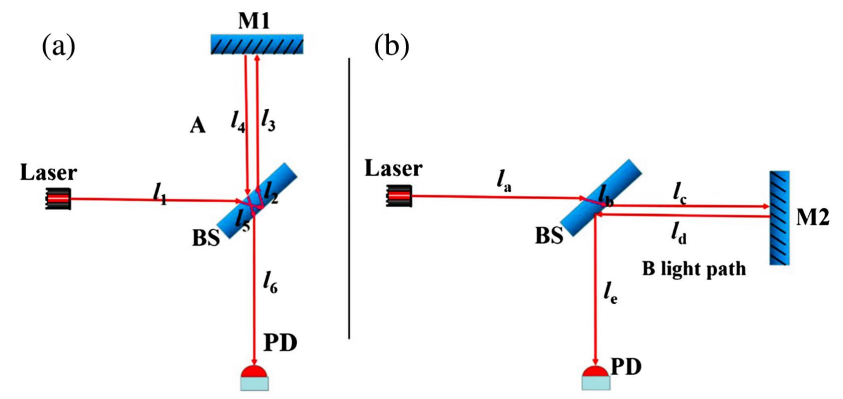

Fig. 2. Two interference light paths [(a) light route A and (b) light route B] separated from the Michelson interferometer. 
Considering that the BS is shaped by temperature, the reflection light route $\left(l_{2}\right)$ and the transmission light route $\left(l_{5}\right)$ in the BS are shown in Fig. 3. The blue line is the light route when the temperature does not change, and the red line represents the path of light after a change in temperature. Where $\theta$ is the incident angle, $\varphi$ is the refractive angle before temperature variation, $\varphi^{\prime}$ is the refractive angle after temperature variation, $L$ is the thickness of the lens, and $\mathrm{d} L$ is a small change in length with temperature.

The first term on the right side of Eq. (3), which indicates the variation coefficient of the reflected optical path with temperature, can be expressed as

$$
\frac{\mathrm{d} l_{2}(n-1)}{\mathrm{d} T}=\frac{2 L}{\sqrt{1-\left(\frac{\sin \theta}{n}\right)^{2}}}\left\{\alpha(n-1)+\frac{\mathrm{d} n}{\mathrm{~d} T}\left[1-\frac{\sin ^{2} \theta(n-1)}{n\left(n^{2}-\sin ^{2} \theta\right)}\right]\right\},
$$

where $\alpha$ is the thermal expansion coefficient, and $\mathrm{d} n / \mathrm{d} T$ is the coefficient of the refractive index with temperature, which depends on the material of lens. Similarly, the second term on the right side of Eq. (3) that expresses the variation coefficient of the transmission optical path with temperature can be expressed as

$\frac{\mathrm{d} l_{5}(n-1)}{\mathrm{d} T}=\frac{L}{\sqrt{1-\left(\frac{\sin \theta}{n}\right)^{2}}}\left\{\alpha(n-1)+\frac{\mathrm{d} n}{\mathrm{~d} T}\left[1-\frac{\sin ^{2} \theta(n-1)}{n\left(n^{2}-\sin ^{2} \theta\right)}\right]\right\}$.

The third term on the right side of Eq. (3) that indicates the thermal effect on the geometric path length can be expressed as

$$
\begin{aligned}
\frac{\mathrm{d} l_{A 0}}{\mathrm{~d} T}= & 3 \frac{\alpha L+L}{\cos \varphi^{\prime}}-\frac{2 \alpha L}{\cos \theta}-\frac{3 L}{\cos \varphi}-\alpha L \\
& +2 \sin \theta\left[\frac{\alpha L \tan \theta}{2}+L \tan \varphi-(\alpha L+L) \tan \varphi^{\prime}\right] \\
& +\left[\alpha L \tan \theta+L \tan \varphi-(\alpha L+L) \tan \varphi^{\prime}\right] .
\end{aligned}
$$
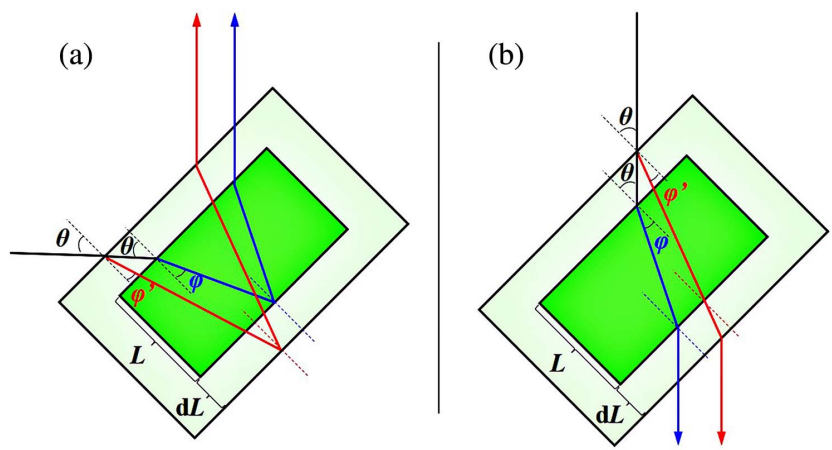

Fig. 3. (a) Reflection optical path and (b) transmission optical path in the BS.
Table 1. The Parameters of Fused Silica Lens in the Interferometer.

Parameters

Value

Thermal expansion coefficient $\alpha$

$0.57 \mathrm{ppm} / \mathrm{K}$

Refractive index variation with temperature $\mathrm{d} n / \mathrm{d} T$

$12.8 \mathrm{ppm} / \mathrm{K}$

Thickness of lens $L$

$7 \mathrm{~mm}$

Substituting Eqs. (4)-(6) into Eq. (3), we will get a complete relationship of the optical path with temperature of light route A. Similarly, the other light route B shown in Fig. 2(b) is analyzed to obtain the coupling coefficient of the optical path with temperature.

To the light route B in Fig. 3(b), the coupling of temperature $\mathrm{d} T$ to the optical path $\mathrm{d} s_{B}$ can be expressed by

$$
\frac{\mathrm{d} s_{B}}{\mathrm{~d} T}=\frac{\mathrm{d} l_{b}(n-1)}{\mathrm{d} T}+\frac{\mathrm{d} l_{B 0}}{\mathrm{~d} T},
$$

where $l_{B 0}$ is the geometric path of light route $\mathrm{B}$. The geometric path of each section is set as $l_{i}(i=a$ to $e$ ). Light route $\mathrm{A}$ and light route $\mathrm{B}$ share the same BS, so $l_{5}$ and $l_{b}$ are the same optical path. The second term on the right side of Eq. (7) can be expressed as

$$
\begin{aligned}
\frac{\mathrm{d} l_{B 0}}{\mathrm{~d} T}= & \frac{\alpha L+L}{\cos \varphi^{\prime}}-\frac{\alpha L}{\cos \theta}-\frac{L}{\cos \varphi}-\frac{\alpha L}{2} \\
& +\sin \theta\left[\alpha L \tan \theta+L \tan \varphi-(\alpha L+L) \tan \varphi^{\prime}\right] .
\end{aligned}
$$

In the end, the variation coefficient of the optical path with temperature can be expressed as

$$
\frac{\mathrm{d} \Delta s}{\mathrm{~d} T}=\frac{\mathrm{d} s_{A}}{\mathrm{~d} T}-\frac{\mathrm{d} s_{B}}{\mathrm{~d} T}=\frac{\mathrm{d} l_{A 0}}{\mathrm{~d} T}-\frac{\mathrm{d} l_{B 0}}{\mathrm{~d} T}+\frac{\mathrm{d} l_{2}(n-1)}{\mathrm{d} T} .
$$

Equations (2)-(9) show the theoretical calculation method for the temperature coupling coefficient of the basic Michelson interferometer. The fused silica is set as the lens material. The parameters of the lens in the interferometer are shown in Table 1. After the calculation, the coefficient of the optical path with temperature would be $215.3 \mathrm{~nm} / \mathrm{K}$. In space-based GWs detection, the displacement measurement noise needs to be $1 \mathrm{pm} / \mathrm{Hz}^{1 / 2}$, so the temperature noise needs to be lower than $4.6 \mu \mathrm{K} / \mathrm{Hz}^{1 / 2}$, which is difficult to obtain in space. Therefore, it is necessary to optimize the optical path.

\section{The Method of Temperature Compensation}

In the interferometer with an ordinary light source, the coherence length of the light is short, and the optical path needs to be compensated by a compensator (as shown in Fig. 4). In laser interferometers, the coherence length of the laser is longer, so the compensator becomes insignificant. However, bonding 


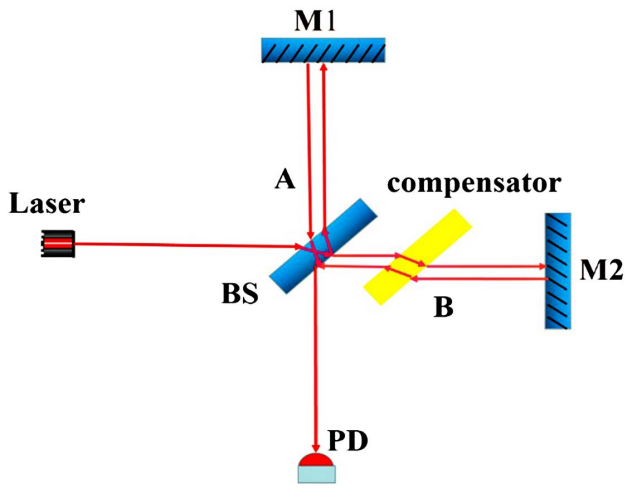

Fig. 4. Compensator in the Michelson interferometer.

the compensator and the lens into an integrated ultra-stable OBI with the same material can change the temperature coupling coefficient. Therefore, we can use the compensator to suppress the thermal effect.

After the compensator is added to the light route B, by using the calculation method in Section 2, Eq. (7) can be modified as

$$
\frac{\mathrm{d} s_{B}}{\mathrm{~d} T}=\frac{\mathrm{d} l_{b}(n-1)}{\mathrm{d} T}+\frac{\mathrm{d} l_{B 0}}{\mathrm{~d} T}+\frac{\mathrm{d} l_{s 1}(n-1)}{\mathrm{d} T}+\frac{\mathrm{d} l_{s 2}(n-1)}{\mathrm{d} T} .
$$

$l_{s 1}$ and $l_{s 2}$ are the optical paths that pass through the compensator twice. They are equal to each other in terms of $l_{s}$, so the variation coefficient in Eq. (9) can be modified as

$$
\frac{\mathrm{d} \Delta s}{\mathrm{~d} T}=\frac{\mathrm{d} s_{A}}{\mathrm{~d} T}-\frac{\mathrm{d} s_{B}}{\mathrm{~d} T}=\frac{\mathrm{d} l_{A 0}}{\mathrm{~d} T}-\frac{\mathrm{d} l_{B 0}}{\mathrm{~d} T}+\frac{\mathrm{d} l_{2}(n-1)}{\mathrm{d} T}-2 \frac{\mathrm{d} l_{s}(n-1)}{\mathrm{d} T} .
$$

Because $l_{s 1}$ and $l_{s 2}$ are for transmission, as Fig. 3(b), it is similar to Eq. (5):

$$
\begin{aligned}
& \frac{\mathrm{d} l_{s}(n-1)}{\mathrm{d} T} \\
& =\frac{\mathrm{d} l_{s 1}(n-1)}{\mathrm{d} T}=\frac{\mathrm{d} l_{s 1}(n-1)}{\mathrm{d} T} \\
& =\frac{L_{s}}{\sqrt{1-\left(\frac{\sin \theta_{s}}{n}\right)^{2}}}\left\{\alpha(n-1)+\frac{\mathrm{d} n}{\mathrm{~d} T}\left[1-\frac{\sin ^{2} \theta_{s}(n-1)}{n\left(n^{2}-\sin ^{2} \theta_{s}\right)}\right]\right\},
\end{aligned}
$$

where $L_{s}$ is the thickness of the compensator, and $\theta_{s}$ is the incident angle of the compensator injected by the laser.

In combination with Eqs. (4), (6), (8), (11), and (12), it can be indicated that the coupling coefficient of the optical path and temperature is related to $L_{s}$ and $\theta_{s}$ of the compensator. In the case of different thickness $L_{s}$ and different incident angle $\theta_{s}$ of the compensator, we can, respectively, get the theoretical model of the variation of the optical path with temperature in Fig. 5. In order to verify the results more reliably, the finite element analysis software of COMSOL Multiphysics is used to analyze the coupling of the OBI optical field and temperature field. In Fig. 5, the initial thickness of the compensator is also set as $7 \mathrm{~mm}$, and
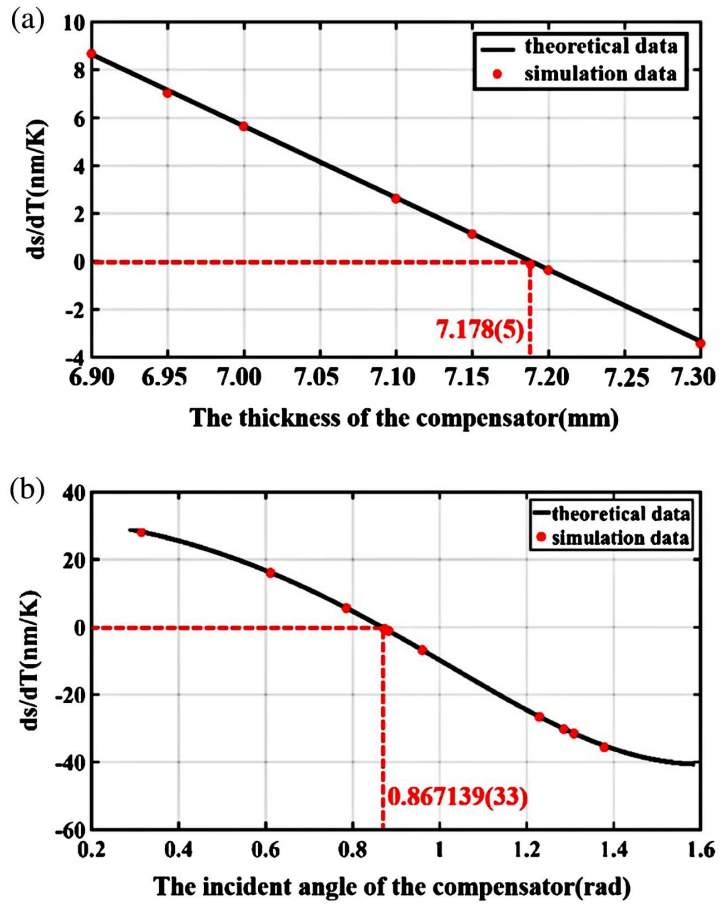

Fig. 5. (a) Coefficient of the optical path variation with temperature when the incident angle $\theta_{s}$ is constant and the thickness $L_{s}$ is variable. (b) Coefficient of the optical path variation with temperature when the thickness $L_{s}$ is constant and the incident angle $\theta_{s}$ is variable.

the incident angle of the compensator is set as $45 \mathrm{deg}(\pi / 4 \mathrm{rad})$; other parameters are from Table 1 . The black lines represent the calculation results of the theoretical model, and the red dots represent the simulation results of COMSOL of the discrete sets of specific parameters. As a result, the theoretical model calculation and the finite element simulation results are consistent.

In this method of temperature compensation, two variables can be used as adjustments. Actually, the processing accuracy of the lens is about $5 \mu \mathrm{m}$, so changing the thickness can just achieve a coupling coefficient of $10 \mathrm{pm} / \mathrm{K}$ at most when the thickness is $7.178 \mathrm{~mm}$. The angle adjustment error can reach $33 \mu \mathrm{rad}$, so the coupling coefficient can be reduced to $2 \mathrm{pm} / \mathrm{K}$ when the incident angle of the compensator is $0.867139(33) \mathrm{rad}$ [about 49.683(2) deg]. For the convenience in application, the method of changing the incident angle is preferable for optimization. As an optimization method, it can also be extended to the optical path design of more complex laser interferometers.

\section{Temperature Compensation Design for the Laser Interferometer in the LPF}

The high precision laser interferometer of ESA's LPF (launched on December 3, 2015, ended on June 30,2017) consists of four different Mach-Zehnder heterodyne interferometers to achieve a differential acceleration noise between its two test masses ${ }^{[31,32]}$. Referring to the design for measuring the single test mass of LPF, we designed a high precision heterodyne laser interferometer to 
verify the temperature compensation method. The OBI of it is shown in Fig. 6.

In the OBI of Fig. 6 , light route $\mathrm{A}$ and light route $\mathrm{B}$ are obtained by Laser 1 , and light route $\mathrm{C}$ and light route $\mathrm{D}$ are obtained by Laser 2. Light A and light D interfered at PD1 to measure the displacement of the test mass, and light $\mathrm{B}$ and light $\mathrm{C}$ interfered at PD2 as a reference signal. By the difference of the

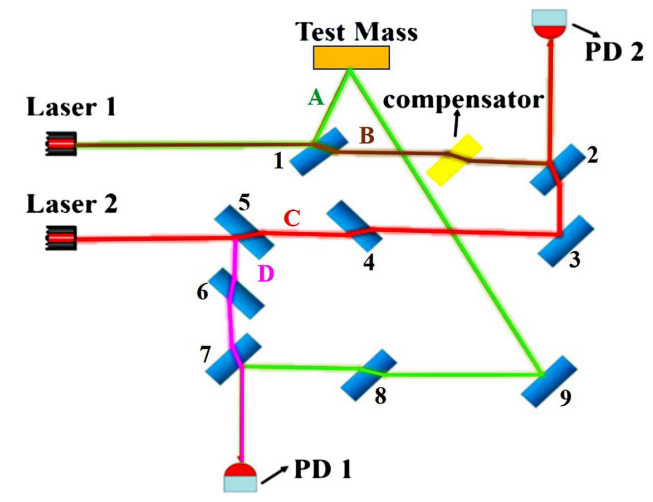

Fig. 6. Optical path of the heterodyne laser interferometer, which refers to LPF, is designed with a compensator. Laser 1 and Laser 2 are the heterodyne laser sources obtained by acousto-optical modulators (AOMs).

Table 2. The Incident Angle of Each Lens of the OBI.

\begin{tabular}{lc}
\hline Lens No. (in Fig. 6) & Incident Angle \\
\hline $2,3,4,5,6,7,8$ & $45^{\circ}$ \\
1 & $55^{\circ}$ \\
9 & $35^{\circ}$ \\
Compensator & $45^{\circ}$ (initial) \\
\hline
\end{tabular}

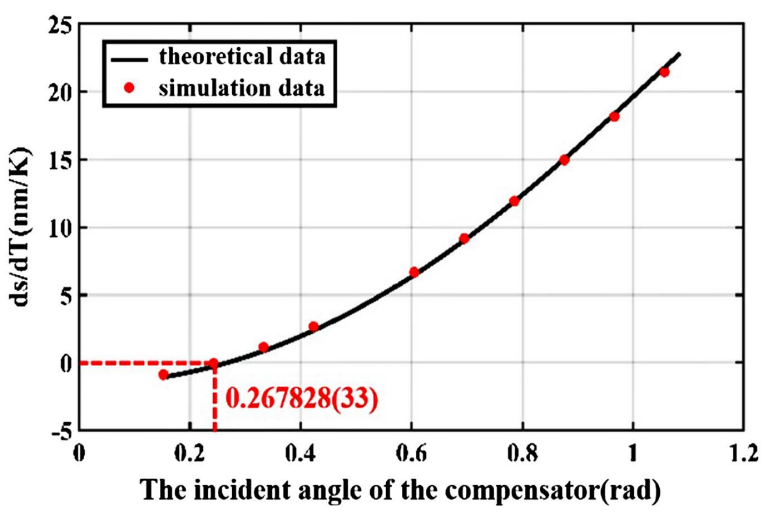

Fig. 7. Relationship of the temperature and optical coupling coefficient and the incident angle of the compensator. The black line is the result of the theoretical model, and the red dots are the results of the COMSOL simulation. two interference signals, the test mass displacement can be obtained, and the common mode noise of the laser source can be suppressed. The lenses in the OBI have the same parameters as in Table 1, and the incident angle of each lens is shown in Table 2.

According to the analysis results in Section 3, both thickness and incident angle will affect the temperature-optical path coupling coefficient of the OBI. Thickness is difficult to control, so we set the thickness as a constant of $7 \mathrm{~mm}$. The relationship between the temperature coupling coefficient and incident angle is shown in Fig. 7. As a result, when the incident angle of the compensator is set as $0.267828 \mathrm{rad}$ (about $15.345 \mathrm{deg}$ ), the temperature coupling coefficient will be better than $1 \mathrm{pm} / \mathrm{K}$.

\section{Conclusions}

We present an effective compensation method of the thermal effect that can be used in an ultra-stable laser interferometer, especially for laser interferometry in space-based GW detectors. The thickness and incident angle of a compensator installed at a specified position along the optical path of the interferometer can affect the temperature coupling coefficient of the whole OBI. By establishing a theoretical model and simulating with the finite element analysis for the laser interferometer of LPF, we obtain an optimized design of a compensator with a thickness of $7 \mathrm{~mm}$ and an incident angle of $0.267828 \mathrm{rad}$, which makes the temperature coupling coefficient of the laser interferometer better than $1 \mathrm{pm} / \mathrm{K}$. We expect that this method will be very useful for the precision laser interferometry in harsh space environment.

\section{Acknowledgement}

This work was supported by the Natural Science Foundation of Guangdong Province (No. 2021A1515010198), the Guangzhou Science and Technology Plan Project (No. 202102020794), and the National Key R\&D Program of China (No. 2020YFC2200500).

\section{References}

1. M. Bailes, B. K. Berger, P. R. Brady, M. Branchesi, K. Danzmann, M. Evans, K. Holley-Bockelmann, B. R. Iyer, T. Kajita, S. Katsanevas, M. Kramer, A. Lazzarini, L. Lehner, G. Losurdo, H. Lück, D. E. McClelland, M. A. McLaughlin, M. Punturo, S. Ransom, S. Raychaudhury, D. H. Reitze, F. Ricci, S. Rowan, Y. Saito, G. H. Sanders, B. S. Sathyaprakash, B. F. Schutz, A. Sesana, H. Shinkai, D. H. Shoemaker, J. Thorpe, J. F. J. van den Brand, and S. Vitale, "Gravitational-wave physics and astronomy in the 2020s and 2030s," Nat. Rev. Phys. 3, 344 (2021).

2. H. Bondi, M. Burg, and A. Metzner, "Gravitational waves in general relativity," Nature 186, 535 (1960).

3. J. Miller, L. Barsotti, S. Vitale, P. Fritschel, M. Evans, and D. Sigg, "Prospects for doubling the range of advanced LIGO," Phys. Rev. D 90, 062005 (2015).

4. V. Connaughton, E. Burns, A. Goldstein, L. Blackburn, M. S. Briggs, B.-B. Zhang, J. Camp, N. Christensen, C. M. Hui, P. Jenke, T. Littenberg, J. E. McEnery, J. Racusin, P. Shawhan, L. Singer, J. Veitch, C. A. WilsonHodge, P. N. Bhat, E. Bissaldi, W. Cleveland, G. Fitzpatrick, M. M. Giles, 
M. H. Gibby, A. von Kienlin, R. M. Kippen, S. McBreen, B. Mailyan, C. A. Meegan, W. S. Paciesas, R. D. Preece, O. J. Roberts, L. Sparke, M. Stanbro, K. Toelge, and P. Veres, "Fermi GBM observations of LIGO gravitational wave event GW150914," Astrophys. J. Lett. 6, 826 (2016).

5. B. C. Barish, "The laser interferometer gravitational-wave observatory LIGO,” Adv. Space Res. 25, 1165 (2000).

6. R. E. Spero and S. E. Withcomb, "The laser interferometer gravitational-wave observatory (LIGO)," Opt. Photon. News 6, 35 (1995).

7. K. Danzmann, "LISA-Laser Interferometer Space Antenna for gravitational wave measurements," Class. Quantum Grav. 13, A247 (2000).

8. S. Babak, J. Gair, A. Sesana, E. Barausse, C. F. Sopuerta, C. P. L. Berry, E. Berti, P. Amaro-Seoane, A. Petiteau, and A. Klein, "Science with the space-based interferometer LISA. V. Extreme mass-ratio inspirals," Phys. Rev. D 95, 103012 (2017).

9. P. Auclair, J. J. Blanco-Pillado, D. G. Figueroa, A. C. Jenkins, M. Lewicki, M. Sakellariadou, S. Sanidas, L. Sousa, D. A. Steer, J. M. Wachter, and S. Kuroyanagi, "Probing the gravitational wave background from cosmic strings with LISA," J. Cosmol. Astropart. Phys. 4, 034 (2020).

10. M. Lau, I. Mandel, A. Vigna-Gómez, C. J. Neijssel, S. Stevenson, and A. Sesana, "Detecting double neutron stars with LISA," Mon. Not. R. Astron. Soc. 3, 492 (2020)

11. T. J. Sumner and D. Shaul, "The observation of gravitational waves from space using LISA,” Mod. Phys. Lett. A 19, 785 (2008).

12. J. Luo, L. S. Chen, H. Z. Duan, Y. G. Gong, S. C. Hu, J. H. Ji, Q. Liu, J. W. Mei, V. Milyukov, M. Sazhin, C. G. Shao, V. T. Toth, H. B. Tu, Y. M. Wang, Y. Wang, H. C. Yeh, M. S. Zhan, Y. H. Zhang, V. Zharov, and Z. B. Zhou, "TianQin: a space-borne gravitational wave detector," Class. Quant. Grav. 33, 035010 (2015).

13. C. X. Hu, X. H. Li, Y. Wang, W. F. Feng, M. Y. Zhou, Y. M. Hu, S. C. Hu, J. W. Mei, and C. G. Shao, "Fundamentals of the orbit and response for TianQin," Class. Quant. Grav. 35, 095008 (2018).

14. S. L. Huang, X. F. Gong, X. U. Peng, A. S. Pau, X. Bian, Y. W. Chen, X. Chen, Z. Fang, X. F. Feng, F. K. Liu, S. Li, X. Li, Z. R. Luo, M. X. Shao, S. Rainer, W. L. Tang, Y. Wang, Y. Wang, Y. L. Zang, and Y. K. Lau, "Gravitational wave detection in space-a new window in astronomy," Scientia Sinica 47, 010404 (2017).

15. W. R. Hu and Y. L. Wu, "The Taiji Program in space for gravitational wave physics and the nature of gravity," Natl. Sci. Rev. 4, 685 (2017).

16. B. Caron, S. Bellucci, F. L. Diberder, A. Dominjon, C. Drezen, R. Flaminio, X. Grave, F. Marion, L. Massonnet, C. Mehmel, R. Morand, B. Mours, M. Yvert, D. Babusci, S. Bellucci, G. Candusso, G. Giordano, G. Matone, L. Dognin, J. M. Mackowski, M. Napolitano, L. Pinard, F. Barone, E. Calloni, L. Di Fiore, A. Grado, L. Milano, G. Russo, S. Solimeno, M. Barsuglia, V. Brisson, F. Cavalier, M. Davier, P. Hello, F. Le Diberder, P. Marin, M. Taubman, F. Bondu, A. Brillet, F. Cleva, H. Heitmann, L. Lattrach, C. N. Man, M. Pham-Tu, J. Y. Vinet, C. Boccara, Ph. Gleyes, V. Loreitte, J. P. Roger, G. Cagnoli, L. Gammaitoni, J. Kovalik, F. Marchesoni, M. Punturo, M. Bernardini, S. Braccini, C. Bradaschia, R. del Fabbro, R. de Salvo, A. Di Virgilio, I. Ferante, F. Fidecaro, A. Gennai, A. Giassi, A. Giazotto, L. E. Holloway, P. La Penna, G. Lorsurdo, F. Palla, H. B. Pan, A. Pasqualetti, R. Pasuello, R. Poggiani, G. Torelli, Z. Zhou, E. Majorana, P. Puppo, P. Rapagnani, and F. Ricci, "Virgo: a laser interferometer for the detection of gravitational waves," J. Instrum. 7, P03012 (2012).

17. Y. Li, Z. Luo, H. Liu, R. H. Gao, and G. Jin, "Laser interferometer for space gravitational waves detection and earth gravity mapping," Microgravity Sci. Technol. 30, 817 (2018).

18. F. Hechler and W. M. Folkner, "Mission analysis for the Laser Interferometer Space Antenna (LISA) mission," Adv. Space. Res. 32, 1277 (2003).

19. J. W. Mei, Y. Z. Bai, J. H. Bao, E. Barausse, L. Cai, E. Canuto, B. Cao, W. M. Chen, Y. Chen, Y. W. Ding, H. Z. Duan, H. M. Fan, W. F. Feng, H. L. Fu, Q. Gao, T. Q. Gao, Y. G. Gong, X. Y. Gou, C. Z. Gu, D. F. Gu, Z. Q. He, M. Hendry, W. Hong, X. C. Hu, Y. M. Hu, Y. X. Hu, S. J. Huang, X. Q. Huang, Q. H. Jiang, Y. Z. Jiang, Y. Jiang, Z. Jiang, H. M. Jin, V. Korol, H. Y. Li, M. Li, M. Li, P. C. Li, R. W. Li, Y. Q. Li, Z. Li, Z. L. Li, Z. X. Li, Y. R. Liang, Z. C. Liang, F. J. Liao, S. Liu, Y. C. Liu, L. Liu, P. B. Liu, X. H. Liu, Y. Liu, X. F. Lu, Y. Lu, Z. H. Lu, Y. Luo, Z. C. Luo, V. Milyukov, M. Ming, X. Y. Pi, C. G. Qin, S. B. Qu, A. Sesana, C. G. Shao, C. F. Shi, W. Su, D. Y. Tan, Y. J. Tan, Z. B. Tan,
L. C. Tu, B. Wang, C. R. Wang, F. B. Wang, G. F. Wang, H. T. Wang, J. Wang, L. J. Wang, P. P. Wang, X. D. Wang, Y. Wang, Y. F. Wang, R. Wei, S. C. Wu, C. Y. Xiao, X. S. Xu, C. Xue, F. C. Yang, L. Yang, M. L. Yang, S. Q. Yang, B. B. Ye, H. C. Yeh, S. H. Yu, D. S. Zhai, C. S. Zhang, H. T. Zhang, J. D. Zhang, J. Zhang, L. H. Zhang, X. Zhang, X. F. Zhang, H. Zhou, M. Y. Zhou, Z. B. Zhou, D. D. Zhu, T. G. Zi, and J. Luo, "The TianQin project: current progress on science and technology," Prog. Theor. Exp. Phys. 5, A107 (2020).

20. Z. Luo, Y. Wang, Y. Wu, W. R. Hu, and G. Jin, "The Taiji program: a concise overview," Prog. Theor. Exp. Phys. 5, A108 (2020).

21. R. Lawrence, M. Zucker, P. Fritschel, P. Marfuta, and D. Shoemaker, "Adaptive thermal compensation of test masses in advanced LIGO," Class. Quant. Grav. 19, 1803 (2001)

22. P. Li, J. Liu, P. Huang, X. Y. Zhang, J. H. Shi, L. B. Yuan, and C. Y. Guan, "Tunable fiber-tip lens based on thermo-optic effect of amorphous silicon," Chin. Opt. Lett. 18, 030602 (2020).

23. J. Luo, Y. Z. Bai, L. Cai, B. Cao, W. M. Chen, Y. Chen, D. C. Cheng, Y. W. Ding, H. Z. Duan, X. Y. Gou, C. Z. Gu, D. F. Gu, Z. Q. He, S. Hu, Y. X. Hu, X. Q. Huang, Q. H. Jiang, Y. Z. Jiang, H. G. Li, H. Y. Li, J. Li, M. Li, Z. Li, Z. X. Li, Y. R. Liang, F. J. Liao, Y. C. Liu, L. Liu, P. B. Liu, X. H. Liu, Y. Liu, X. F. Lu, Y. Luo, J. W. Mei, M. Ming, S. B. Qu, D. Y. Tan, M. Tang, L. C. Tu, C. R. Wang, F. B. Wang, G. F. Wang, J. Wang, L. J. Wang, X. D. Wang, R. Wei, S. C. Wu, C. Y. Xiao, M. Z. Xie, X. S. Xu, L. Yang, M. L. Yang, S. Q. Yang, H. C. Yeh, J. B. Yu, L. H. Zhang, M. H. Zhao, and Z. B. Zhou, "The first round result from the TianQin-1 satellite," Class. Quant. Grav. 37, 185013 (2020).

24. Z. Li, H. Z. Duan, X. Q. Huang, M. Ming, P. B. Liu, S. Zou, G. F. Wang, X. Zhang, M. L. Yang, B. Cao, Q. Liu, S. Q. Yang, L. C. Tu, and H. C. Yeh, "Design and performance test of the spaceborne laser in the TianQin-1 mission," Opt. Laser Technol. 141, 107155 (2021).

25. A. Steimacher, A. N. Medina, A. C. Bento, J. H. Rohling, M. L. Baesso, V. C. S. Reynoso, S. M. Lima, M. N. Petrovich, and D. W. Hewak, "The temperature coefficient of the optical path length as a function of the temperature in different optical glasses," J. Non-Cryst. Solids 348, 240 (2004).

26. A. P. Silva, A. P. Carmo, V. Anjos, M. J. V. Bell, L. R. P. Kassab, and R. D. A. Pinto, "Temperature coefficient of optical path of tellurite glasses doped with gold nanoparticles," Opt. Mater. 34, 239 (2012).

27. M. L. Baesso, J. Shen, and R. D. Snook, "Mode-mismatched thermal lens determination of temperature coefficient of optical path length in soda lime glass at different wavelengths," J. Appl. Phys. 75, 3732 (1994).

28. B. Xie and S. Feng, "Heterodyne detection enhanced by quantum correlation," Chin. Opt. Lett. 19, 072701 (2021).

29. X. Chen, C. Zhou, D. Fan, L. Qian, Y. D. Pang, C. Wei, G. Zhao, S. J. Liang, and Y. X. Li, "Modified frequency-shifted interferometer: encoding wavelength into phase," Chin. Opt. Lett. 18, 101203 (2020).

30. X. F. Wang, L. Feng, P. Chen, Z. Huang, and Y. Yuan, "Micro displacement reconstruction of self-mixing grating interferometer based on Littrow structure," Chin. Opt. Lett. 19, 101402 (2021).

31. M. Armano, H. Audley, J. Baird, P. Binetruy, and P. Zweifel, "Sensor noise in LISA pathfinder: in-flight performance of the optical test mass readout," Phys. Rev. Lett. 126, 131103 (2021).

32. M. Armano, H. Audley, J. Baird, P. Binetruy, M. Born, D. Bortoluzzi, E. Castelli, A. Cavalleri, A. Cesarini, A. M. Cruise, K. Danzmann, M. de Deus Silva, I. Diepholz, G. Dixon, R. Dolesi, L. Ferraioli, V. Ferroni, E. D. Fitzsimons, M. Freschi, L. Gesa, F. Gibert, D. Giardini, R. Giusteri, C. Grimani, J. Grzymisch, I. Harrison, G. Heinzel, M. Hewitson, D. Hollington, D. Hoyland, M. Hueller, H. Inchauspé, O. Jennrich, P. Jetzer, N. Karnesis, B. Kaune, N. Korsakova, C. J. Killow, J. A. Lobo, I. Lloro, L. Liu, J. P. López-Zaragoza, R. Maarschalkerweerd, D. Mance, N. Meshskar, V. Martín, L. Martin-Polo, J. Martino, F. Martin-Porqueras, I. Mateos, P. W. McNamara, J. Mendes, L. Mendes, M. Nofrarias, S. Paczkowski, M. Perreur-Lloyd, A. Petiteau, P. Pivato, E. Plagnol, J. Ramos-Castro, J. Reiche, D. I. Robertson, F. Rivas, G. Russano, J. Slutsky, C. F. Sopuerta, T. Sumner, D. Texier, J. I. Thorpe, D. Vetrugno, S. Vitale, G. Wanner, H. Ward, P. Wass, W. J. Weber, L. Wissel, A. Wittchen, and P. Zweife, "LISA pathfinder performance confirmed in an open-loop configuration: results from the free-fall actuation mode," Phys. Rev. Lett. 123, 111101 (2019). 